\title{
A Modular BLSS Simulation Model
}

John D. Rummel* and Tyler Volk**

*NASA-Ames Research Center, MS 239-4, Moffett Field, CA 94035, U.S.A.

**Department of Applied Science. New York University. New York, NY 10003, U.S.A.

\begin{abstract}
The coordination of material flows in Earth's biosphere is largely made possible by the buffering effect of huge material reservoirs. Without similarly-sized buffers. a bioregenerative life support system (BLSS) for extraterrestrial use will be faced with coordination problems more acute than those in any ecosystem found on earth. A related problem in BLSS design is providing an interface between the various life-support processors, one that will allow for their coordination while still allowing for system expansion. Here we present a modular model of a BLSS that interfaces system processors only with the material storage reservoirs, allowing those reservoirs to act as the principal buffers in the system and thus minimizing difficulties with processor coordination. The modular nature of the model allows independent development of the detailed submodels that exist within the model framework. Using this model. BLSS dynamics were investigated under normal conditions and under various failure modes. Partial and complete failures of various components. such as the waste processor or the plants themselves. drive transient responses in the model system. allowing us to examine the effectiveness of the system reservoirs as buffers. The results from simulations of this sort will help to determine control strategies and BLSS design requirements. An evolved version of this model could be used as an interactive control aid in a future BLSS.
\end{abstract}

\section{INTRODUCTION}

A life support system that uses biological processes to recycle food, air and water holds the ultimate promise of enabling the human race to successfully become established outside the Earth's biosphere. Such a system will also provide an economic advantage for a variety of space missions contemplated for the immediate future /1/. In order to fulfill its promise, a bioregenerative life support system (BLSS) must be safe, reliable. and flexible. Building a BLSS with these qualities will require a detailed knowledge of such systems, so a predictive tool that will allow a myriad of investigations into BLSS design will be useful. In this paper we will present a model that can be used to investigate the workings of a BLSS.

The many problems faced when designing a BLSS have been explained before in this journal $/ 2 /$. so we will not dwell on them. It should be noted, however, that there is an immediate economic pressure to make a BLSS as small as possible, so any near-term BLSS will be considerably different from the life support system that we are all most familiar with. the Earth's biosphere. Thus we will abandon the luxury of the large buffers (e.g.. the oceans and the atmosphere) that play such a significant role in maintaining the Earth as a living planet. This scale change will make it necessary to know as much about the BLSS as a whole as about its component parts.

Other workers have previously used models to study bioregenerative life support systems /3, 4, 5/. These models were often limited to a specific system design. or were aimed specifically at understanding the nature of the control strategies available in a BLSS. Our aim here is to establish an approach to BLSS modelling that can grow along with the changing concepts of BLSS design. We would like to lay a modelling foundation that could be as applicable to an Earth-orbit BLSS as it would be to a system found in a Lunar base. Hence. the model we propose should be viewed as a fluid, hierarchical structure. where changes at one level do not necessarily involve changes at other levels in the model.

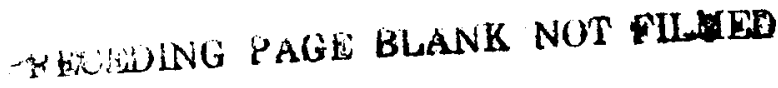




\section{Components of a Bioregenerative Life Support System}

A BLSS can ve said to consist of two basic elements: processors (also know as flow elements or transducers) and storages $/ 5 /$. The processors change the state of materials in the system. and move them from one storage area to another. In the same way that an earth ecosystem is composed of autotrophic and heterotrophic components /6/. a BLSS must contain processors that fix simple inorganic materials into complex substances that are then broken down by the processors that derive energy from them. The storages in a BLSS are equivalent to the vast reservoirs of inorganic materials found on Earth. but because a BLSS will have only a limited amount of reservoir capacity in the system. the level of control needed over the storages in a BLSS will be much greater.

To begin our modelling of a BLSS we defined a system that is made up of five processors and eight storage reservoirs. Only three of the processors have easy biological equivalents in an Earth ecosystem. the Crew and the Waste Processor represent heterotrophic components while the Plants in the system are autotrophs. The Gas Separator(s) and the Nutrient Mixer are simply a means to maintain a greater amount of control over the reservoirs in the system. The storage elements in the system are either mixed storages (the Crew and PGM Atmospheres, the Food Storage. the Waste Storage. and the Nutrient Solution Reservoir) or storages for pure compounds $\left(\mathrm{O}_{2} \cdot \mathrm{CO}_{2} . \mathrm{H}_{2} \mathrm{O}\right.$, and $\left.\mathrm{HNO}_{3}\right)$. These particular reservoirs were chosen to represent the storage types that would most likely be found in an operable BLSS. with the constraint that our model would initially only track the Carbon. Hydrogen. Oxygen. and Nitrogen in the system.
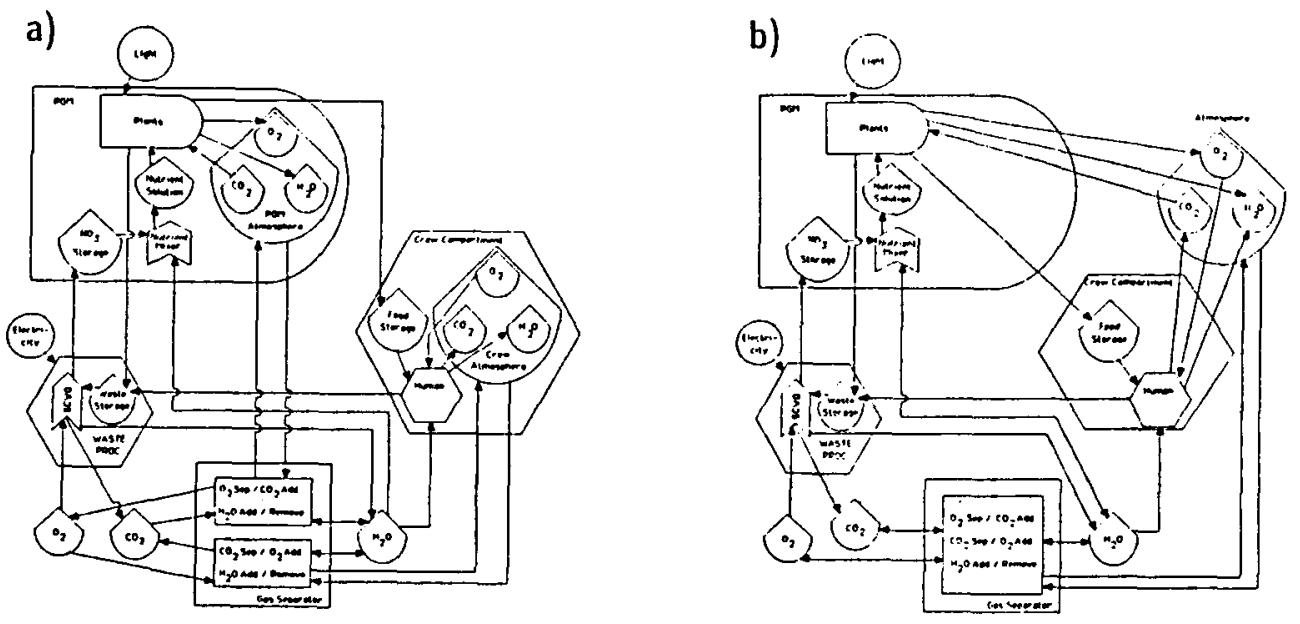

Fig. 1. Two BLSS model structures. In (a) there are two separate atmospheres for the PGM and the crew compartment. In (b) the plants and the people share a common atmosphere.

\section{Structure of the Model}

The principal philosophy that guided the structure of the BLSS presented here is that the system should be modular: i.e., each processor subsystem within the model should be able to work (and be modelled) independently of the others. The corollary condition was therefore established that each processor should interface only with storage reservoirs, and not with other processors. Our adaptation of this philosophy is owed. in part. to conversations held with Columbano /7/ at Ames Research Center.

Though the model is intended to represent the flows and transformations that are seen in a BLSS at the macroscopic level. all such transformations were calculated at the atomic level. Only through this means were we able to maintain the high degree of precision in our calculations (on the order of $10^{9}$ ) needed to conserve the elemental masses represented in the model. Our error level is far below the expected leakage rate of a real bioregenerative system in space. The derivation of the stoichiometry associated with each of the processes in the model is not discussed here. but is given in a companion paper $/ 8 /$. 
The basic structure of two of the BLSS's we studied are shown in Figure 1. The system elements in the figure are portrayed in the energy language of Odum /9/. The BLSS Simulation Model was implemented as a Pascal program running on a VAX 11-785 computer at Ames Research Center. Each of the processors in the system was implemented as a separate Pascal procedure, allowing us to maintain the system's modular design within the program itself. As a result. modifications to the system structure are very easy to make without changing the processors themselves (compare Figure 1a with 1b). A brief description of each of the model BLSS components is given below, along with notes on how they were implemented.

The plants. These processors are found inside of the Plant Growth Module (PGM) shown in Figure 1. For the first iteration of our model we ignored Biblical strictures and postulated that the crew can live on bread alone, so the plants grown are wheat plants. These wheat plants interact with the PGM Atmosphere. where they take up or release $\mathrm{CO}_{2}$ and $\mathrm{O}_{2}$. depending on the lighting conditions. and where they release $\mathrm{H}_{2} \mathrm{O}$ through transpiration. The plants also take up nutrient solution from the Nutrient Solution Reservoir. At harvest time. the edible portion of the wheat is moved to the Food Storage. while the inedible portion of the wheat and the excess edible portion. if any, is sent to the Waste Storage.

The growth of wheat is modelled after data provided to us by Salisbury and Bugbee $/ 10 /$. Because we did not consider temperature variations, wheat growth was postulated to be dependent only on the lighting conditions. and on the concentrations of $\mathrm{CO}_{2}$ and $\mathrm{NO}_{3}$ in the PGM atmosphere. The model for wheat growth is given by the following three equations:

$$
\begin{gathered}
\Delta \text { growth }=\text { inedible mass } \times r \times \frac{\mathrm{V}_{\mathrm{CO}_{2}} \times\left[\mathrm{CO}_{2}\right]}{\mathrm{K}_{\mathrm{CO}_{2}}+\left[\mathrm{CO}_{2}\right]} \times \frac{\mathrm{V}_{\mathrm{NO}_{1}} \times\left[\mathrm{NO}_{3}\right]}{\mathrm{K}_{\mathrm{NO}}+\left[\mathrm{NO}_{3}\right]} \times\left(1-\frac{\text { total mass }}{\text { maximum mass }}\right) \\
\text { edible mass } \text { m }_{1}=\text { edible mass, }+ \text { allocation } \times \Delta \text { growth }
\end{gathered}
$$

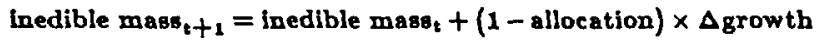

$\mathrm{CO}_{2}$ and $\mathrm{NO}_{3}$ concentrations are related to the total growth rate by the use of Michaelis-Menten kinetics coupled to a logistic growth formulation. using the intrinsic growth rate. r. and a cultivar-specific target size for a single wheat plant. maximum mass (equation (1)). Furthermore. the net growth of each wheat plant derives only from the inedible portion of the plant (leaves. stalks. etc.). Edible portions are assumed to be able to offset their own respiration by photosynthesis. After a preset time the edible and inedible portions of the plant each receive a constant allocation of the production from the inedible mass (equations (2) and (3)). Before that time is reached only the inedible portion of the plant grows.

These equations can represent the growth of a single wheat plant or of a batch of identical wheat plants. In the model, plant growth is tracked on a per-plant basis. Plants start out from seeds taken from the food Storage and are allowed to "germinate" (convert from edible to inedible mass proportions) before being planted in the PGM. The growing period we used for this paper lasted the 55 days from transplanting into the PGM until the harvest of the mature crop.

No doubt some fallacies exist within this model of the plants. but using similar conditions to those reported by Salisbury and Bugbee /10/ the model duplicates very closely the growth of actual wheat plants (Figure 2). Representative results were also gained under sub-optimal conditions and while the "plants" were respiring in the dark. Even so, we do not propose this submodel as a detailed model of a wheat plant. For our immediate purposes. however, it appears to suffice. Future BLSS model development will pay careful attention to improvements in the plant submodels employed.

The nutrient mixer. This processor is also located in the PGM. Its simple function is to refill the Nutrient Solution Reservoir with water and with nitrate (in the form of nitric acid). Thus it interfaces with the $\mathrm{HNO}_{3}$ Reservoir and with the $\mathrm{H}_{2} \mathrm{O}$ Reservoir. First it refills the Nutrient Solution Reservoir with the proper amount of water, and then it adds enough nitric acid to maintsin the reservoir at the proper nitrate setpoint. 


\section{Wheat Growth Curve - Yecora Rojo \\ Model vs. Data, 1200 plants}

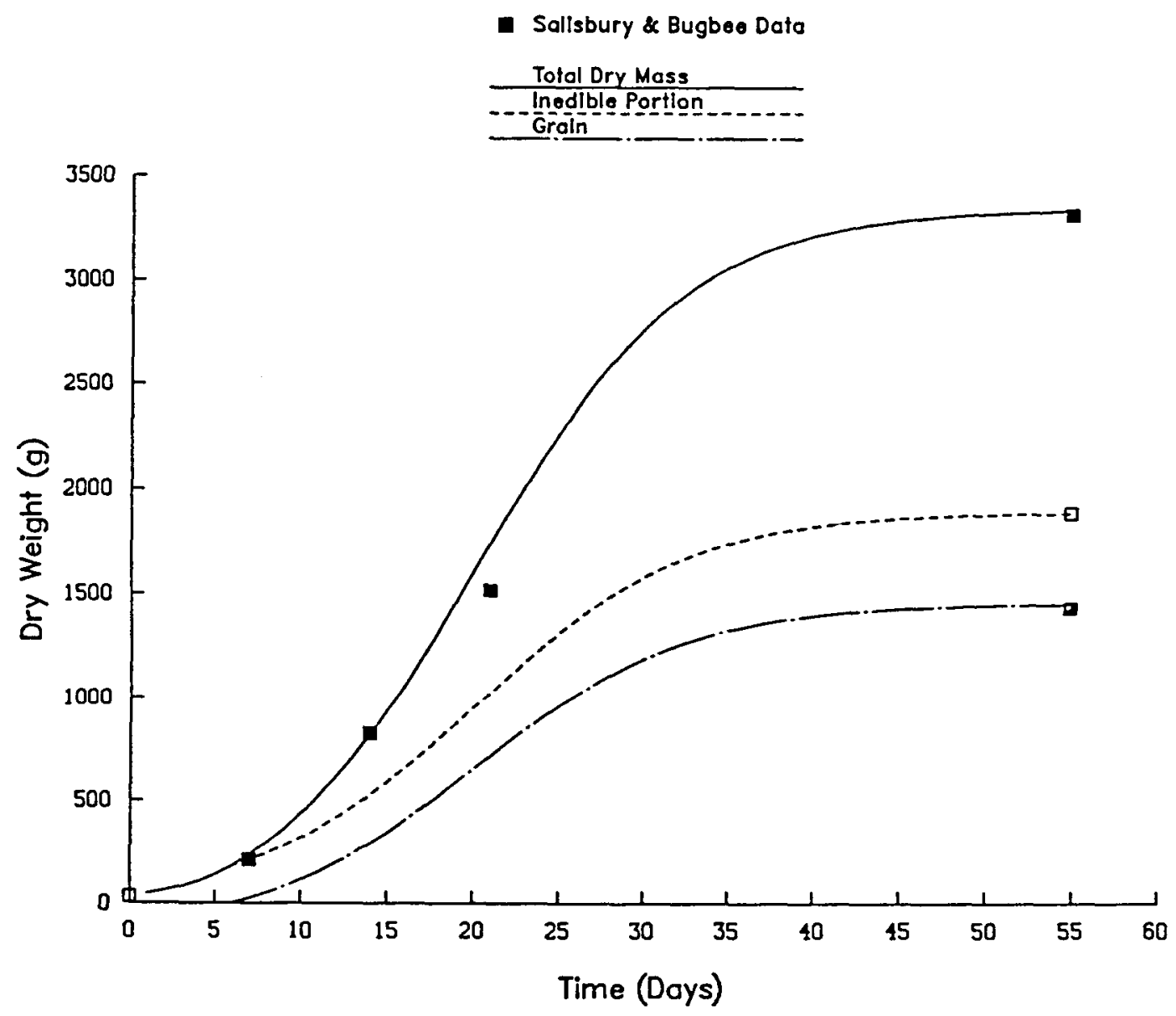

Fig. 2. Wheat growth in $1 \mathrm{~m}^{2}$ from both actual data and the plant-growth submodel used in the BLSS Simulation Model. Growing conditions in the model were constant light. $1000 \mu \mathrm{l} / 1$ $\mathrm{CO}_{2}$, and $4.0 \mathrm{mM} \mathrm{NO}_{3}^{-}$.

The crew. The Crew lives in the crew module. eat harvested food from the Plants (found in Food Storage). and drink water from the $\mathrm{H}_{2} \mathrm{O}$ Reservoir. After the food is metabolized, the Crew adds human waste and waste water to the Waste Storage. Crewmembers typically exchange $\mathrm{CO}_{2}, \mathrm{H}_{2} \mathrm{O}$ vapor, and $\mathrm{O}_{2}$ with the Crew Atmosphere. The crewmembers can have individual sleeping and eating schedules. Each person eats three meals a day and uses drinking water only while awake. Wash water is used over the entire 24 hour day. All crewmembers are assumed to have identical metabolisms. with no metabolic provision being made for a net change in the mass of the Crew. The life support needs of the crewmembers were gleaned from a number of different sources $/ 11$. 12. 13/. but where necessary. a conservative estimate was taken over a more stringent one. The mass of the materials used by each crewman per day are given in Table 1.

The waste processor. This processor acts to break down waste materials into their useful inorganic components. The waste processor takes in material from the Waste Storage and oxygen from the $\mathrm{O}_{2}$ Reservoir, and returns water to the $\mathrm{H}_{2} \mathrm{O}$ Reservoir. carbon dioxide to the $\mathrm{CO}_{2}$ Reservoir. and nitric acid to the $\mathrm{HNO}_{3}$ Reservoir. Our conception of the waste processor is based on Super Critical Wet Oxidation /14/. but a number of other processes are also available $/ 15 /$. We have only implemented one level of waste processing in the current version of the model. with all wastes going through the same processor. Perhaps the most blatant simplification in this first version of our BLSS simulation model is that the waste processor converts $100 \%$ of the wastes into useable substances. This includes the recapture of all of the nitrogen as nitrate. One of the first improvements we will make in the model will be to add in a realistic level of denitrification. both in the waste processor and in the plant growth module nutrient solution. 
TABLE 1 BLSS Crew Mass Flows (g/person/day)

Inputs

$\mathrm{H}_{2} \mathrm{O}$ :

Drinking/Food Preparation Water

4577.3

Water in Food

Wash/Flush Water

18000.0

Food:

Edible Dry Wheat

$\mathrm{O}_{2}$ :

To Metabolize Wheat

804.6

Outputs

$\mathrm{H}_{2} \mathrm{O}$ :

Water in Urine. Feces

3025.5

Metabolic Water (Vapor)

406.0

Perspiration Water (Vapor)

1680.0

Wash/Flush Water

18000.0

Solids:

Feces, Urine, Sweat Solids

161.4

$\mathrm{CO}_{2}$ :

From Metabolized Wheat

1092.3

The gas separator. In order to maintain atmospheric gases at their setpoints. the gas separator adds or removes $\mathrm{O}_{2} . \mathrm{CO}_{2}$. and $\mathrm{H}_{2} \mathrm{O}$ vapor from the $\mathrm{PGM}$ or $\mathrm{Crew}$ Atmosphere. These gases are supplied from or returned to their respective reservoirs. No specific technology was envisioned for this apparatus. Future investigations with the model will also consider a system without such a device.

The system reservoirs. In the model each reservoir is implemented as either a complex (for the mixed storages) or a simple Pascal variable. In a real system the reservoirs would consist of a storage tank (or a pressure hull in the case of the atmospheres) and the accompanying distribution piping leading to it. As such. each reservoir can be thought of as a "bus" to which the appropriate processors can be attached.

\section{SAMPLE RESULTS}

In order to demonstrate the Modular BLSS Simulation Model. a number of runs were undertaken. Each of the runs discussed below were based on the same basic system conditions. A crew of 6 was specified, living in a $280 \mathrm{~m}^{3}$ atmosphere. For the crew the air temperature was $20^{\circ}$, the relative humidity $50 \%$. the $\mathrm{O}_{2}$ level $20 \%$. and the $\mathrm{CO}_{2}$ level set at $350 \mu \mathrm{l} / \mathrm{l}$. Food and air consumption for the crew is listed in Table 1.

All crop plantings were based on providing slightly more than $855 \mathrm{~g} /$ person/day over the 55 days from planting to harvest. As a result. a total of 247.500 wheat plants were grown in each run. to provide an estimated production surplus of $42.5 \mathrm{~g} /$ person/day. The atmospheric conditions were the same for the plants and the crew, with the exceptions that the PGM relative humidity was maintained at $70 \%$. and the $\mathrm{CO}_{2}$ level was maintained at 1000 $\mu \mathrm{l} / \mathrm{l}$. Lighting for the plants was maintained for $24 \mathrm{hrs} /$ day.

The waste processor also ran for $24 \mathrm{hrs} /$ day (to minimize preheat penalty). with a maximum capacity of 8.5 $\mathrm{kg} / \mathrm{hr}$ wet waste. This value was sufficient to meet the total daily needs of the system. plus a small excess capacity. 
The pure storage reservoirs were initially filled with arbitrarily high levels of materials. enough to guarantee sufficient supplies throughout the fluctuations of a normal year in the BLSS. For the runs below. the $\mathrm{H}_{2} \mathrm{O}$ Reservoir initiaily held $10.000 \mathrm{~kg}$. the $\mathrm{CO}_{2}$ reservoir held $6.000 \mathrm{~kg}$. and the $\mathrm{O}_{2}$ and $\mathrm{HNO}_{3}$ reservoirs were each filled with $1.000 \mathrm{~kg}$. By tracing the maximum and minimum levels reached by a reservoir in any run. the necessary reservoir capacities could be determined by reference to these initial starting levels. At the beginning of all runs the waste reservoir was empty. and the atmospheres and the Nutrient Reservoir were at there respective setpoints. An initial food supply of $340 \mathrm{~kg}$ dry wheat was also provided. both as a food reserve and for use as seeds $(7.4 \mathrm{~kg})$.
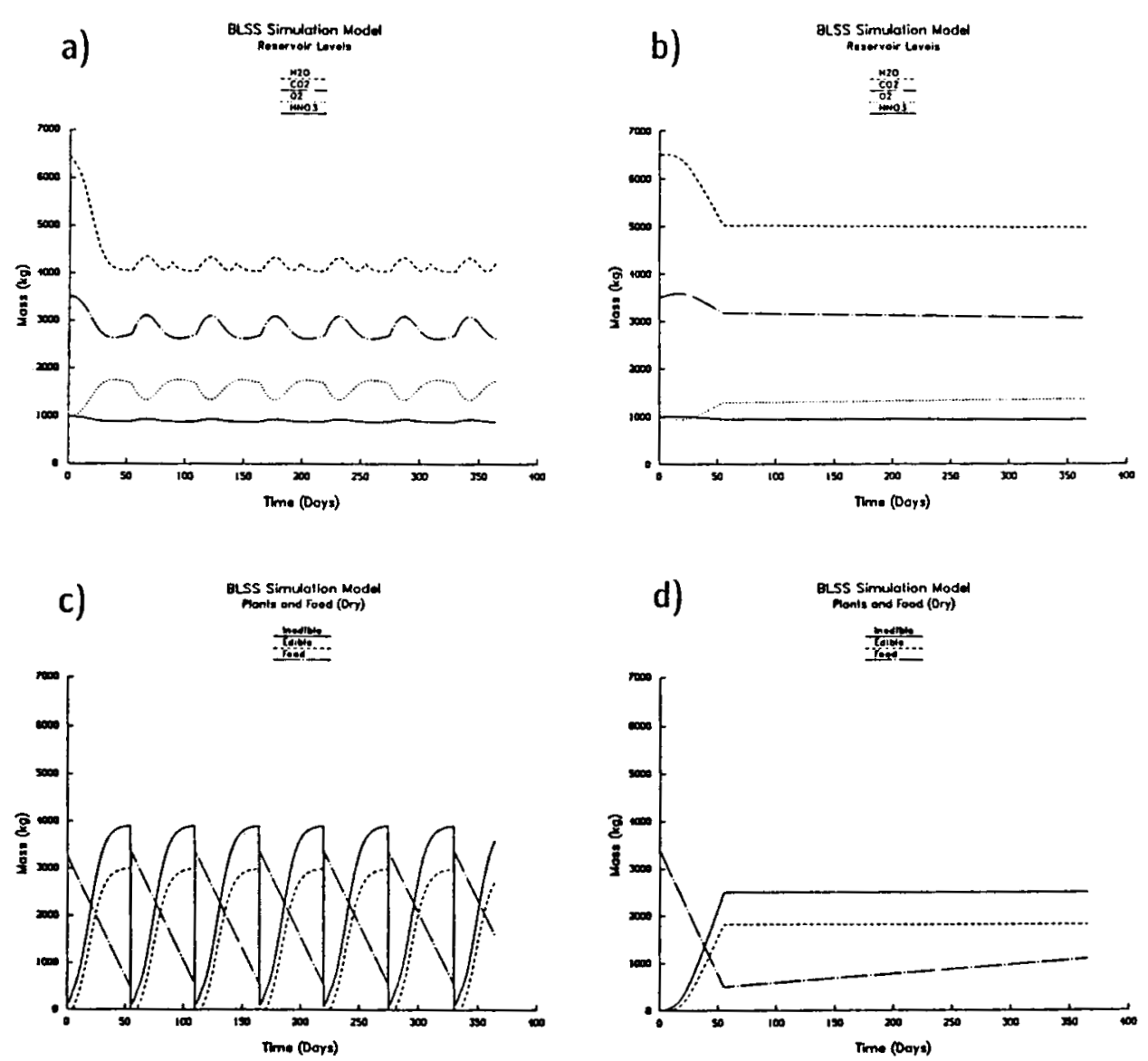

Fig. 3. Results from two planting schemes. Planting one big batch èvery 55 days results in the graphs shown in (a) for the reservoir levels. and (c) for the dry plant biomass in the systern. These are contrasted to the results gained from planting and harvesting one batch per day (b and d). The food storage level in (d) eventually reaches the initial amount during the fifth year of system operation. 

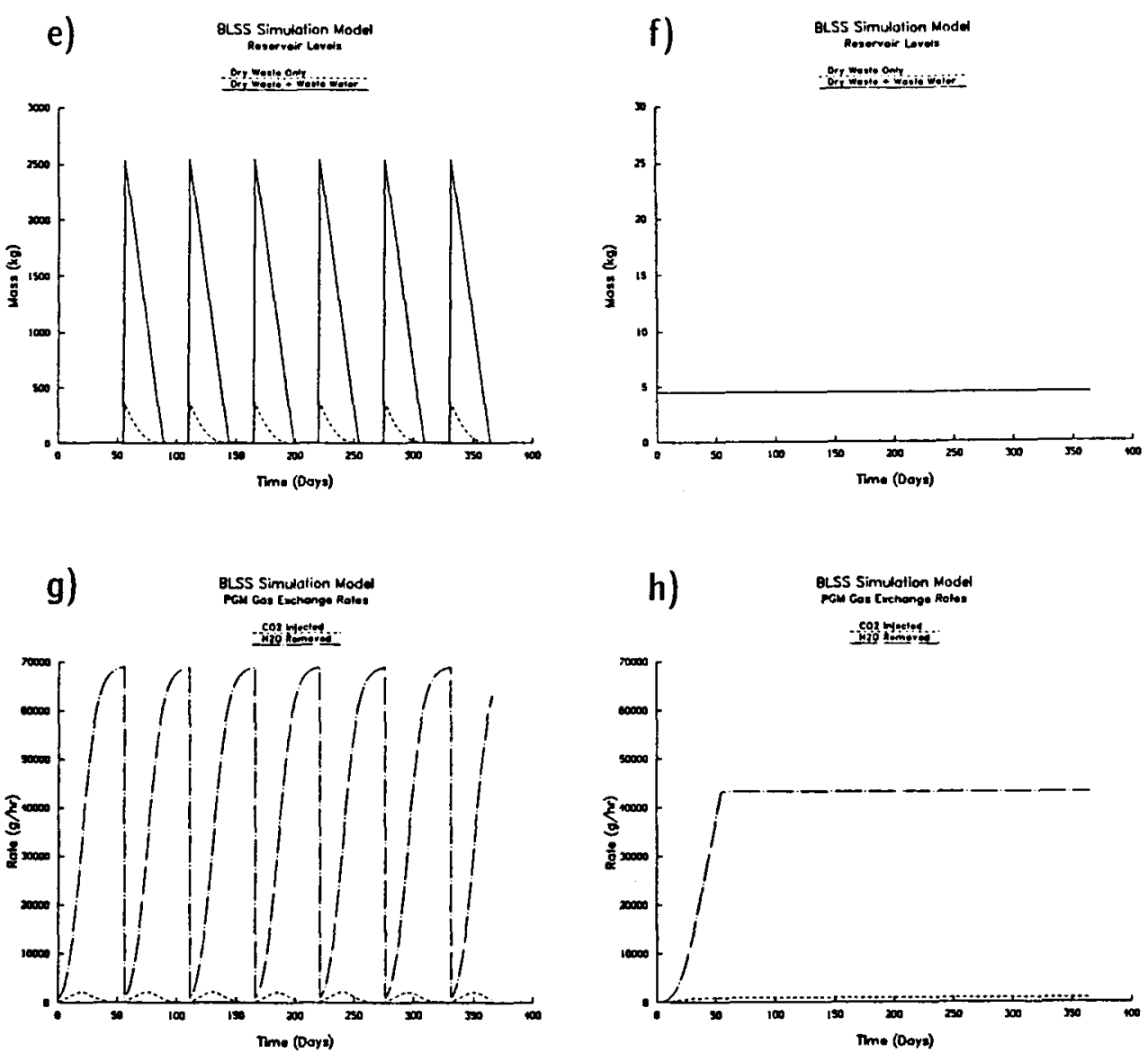

Fig. 3 (cont.) Results from one-big-batch planting are shown in (e) for the waste reservoir and in (g) for the hourly gas exchange rates. Contrasting results from the one-batch-per-day scheme are shown in (f) and (h). Note the scale difference between (e) and (f).

\section{Planting Considerations}

Two planting methods were compared. The first involved planting all 247.500 plants in one big batch. beginning on the first day and repeating the process after every harvest. The second method involved making daily plantings of 4500 plants, and after 55 days making one harvest and one planting each day. This small batch method therefore used the same number of plants as the big batch method.

The effects of planting one big batch versus 55 small batches can be seen in Figure 3 . It is clear that having a daily harvest/planting cycle results in a much more stable situation in the system reservoirs. as well as in the $\mathrm{CO}_{2}$ injection and water condensation apparatus. In fact. in the variant of the model with the PGM and $\mathrm{Crew}$ Atmosphere connected (Figure $1 \mathrm{~b}$ ). the small batch system only requires the addition of $\mathrm{CO}_{2}$ into the single atmosphere. Even with that connection the big batch method would require both addition and removal of $\mathrm{CO}_{2}$. Hence the mass penalty for $\mathrm{CO}_{2}$ removal equipment would not have to be paid in such a system if the correct planting scheme is adopted. 
Babcock et al. /4/ used a 10-day waste processor failure to investigate the dynamic interaction of system mass and storage sizes in a simple CELSS model. Figure 4 compares the effects of such a failure in a BLSS with one big batch planting to the effects in a BLSS with daily plantings. The severity of the effects of this failure on the big batch planting varies depending on the timing of the failure. It is obviously worse if the failure occurs during harvest time. This consideration does not affect the system with daily plantings/harvests. Because the storage reservoirs in these runs have a good deal of excess capacity the plants and the crew are unaffected by the waste processor failures shown here. During the failure the system lowered the level of the $\mathrm{H}_{2} \mathrm{O}$ Reservoir by almost $2.000 \mathrm{~kg}$ more than is normal with the big batch planting. and by more than $1.500 \mathrm{~kg}$ when the daily planting scheme was used.

\section{Crop Failure}

We have also investigated the system under both planting schemes when all or a portion of the crop fails. As with the waste processor failure, in case of a crop failure the scheme with one big batch planting is much more sensitive to the timing of the failure than is the scheme with daily plantings. If the single large crop is destroyed right before harvest it is much more devastating than if a young crop is destroyed. Having the small daily plantings puts a smaller proportion of the overall crop at risk at any one time. After the BLSS has reached equilibrium with the daily planting scheme. destroying the whole crop is equivalent to a restart of the system. No shortages will take place. With the single big batch planting scheme. crop failures at certain times (e.g.. at 45 days after crop planting) will cause the system to run out of food.
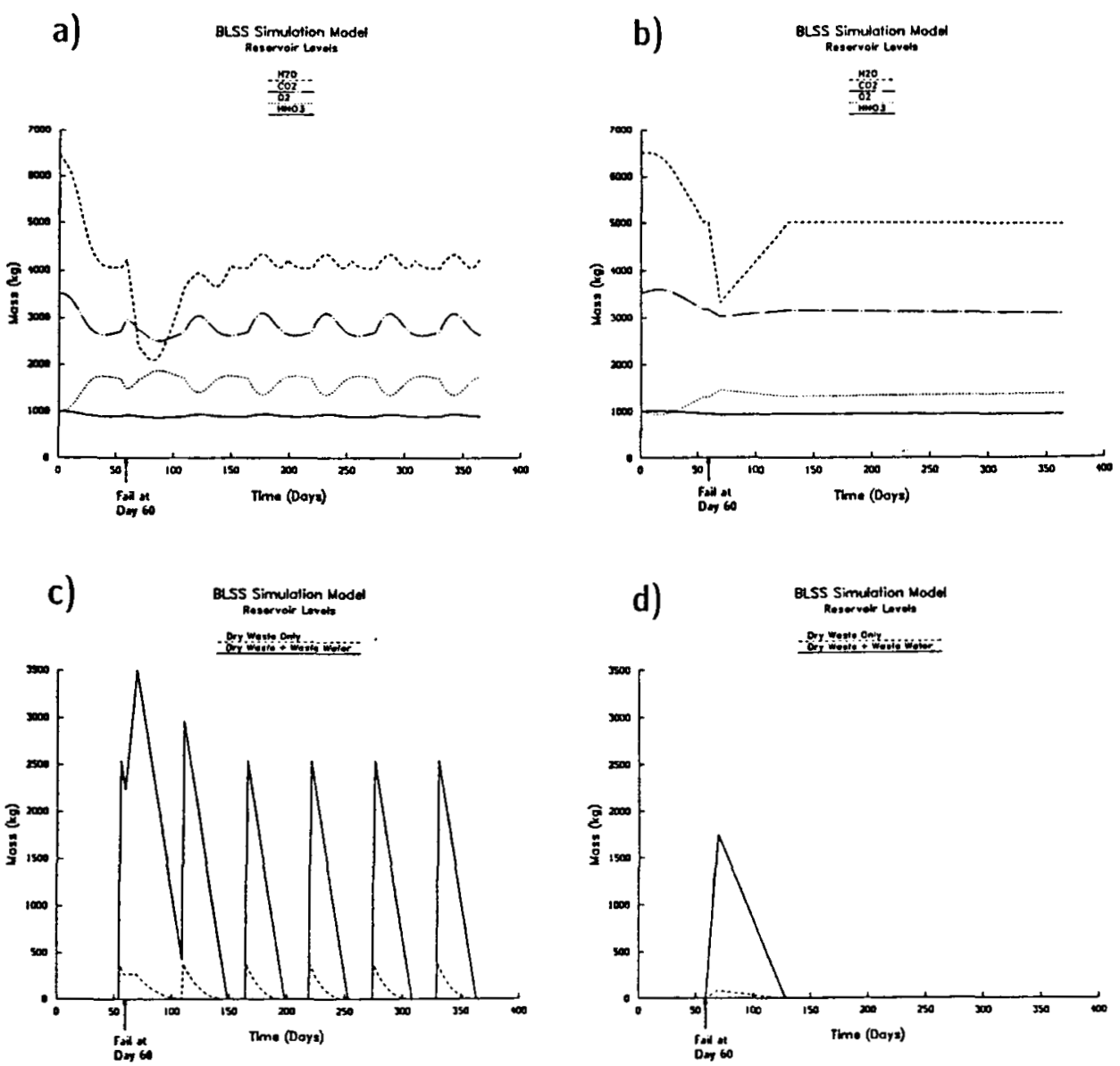

Fig. 4. Waste processor failure. The waste processor was failed at day 60 and restarted at day 70. Reservoir levels from the one-big-batch planting scheme are shown in (a) and (c). Results from the one-batch-per-day scheme are shown in (b) and (d). 


\section{DISCUSSION}

One fact that is apparent in the results of the Modular BLSS Simulation Model is that such a system can be designed to be stable with only a minimum amount of dynamic control on the system processors. Control of the system is strongly dependent on control of the plants and the waste processor. If those processors remain stable and are interfaced with sufficient material buffers. then the system itself can remain stable over long periods.

Because processor stability is maintained. it is also apparent that a planting scheme that maintains a nearly constant amount of growing biomass will be more successful than the alternate scheme of large batch cultivation. This difference is maintained even under various failure modes that affect the BLSS. While common sense might also point to this conclusion, the model presented here allows us to quantify the advantages of the one scheme over the other.

The BLSS Simulation Model described here can be of use in investigating additional system. To improve the usefulness of the model in assessing different aspects of BLSS design. the model will be modified and improved to meet those needs. As was mentioned above. the model will soon be expanded to include nitrogen cycling and the effects of partial waste-processor returns on the system mass flows. In addition, the plant and crew submodels will be improved to improve our investigations into the reservoir-size boundary conditions. Eventually the scope of the model will be expanded to track other elements. including P. S. and an array of micronutrients such as K. Ca. and $\mathrm{Mg}$.

\section{REFERENCES}

1. R.L. Olson, E.A. Gustan, and T.J. Vinopal. CELSS transportation analysis. Adv. Space Res. 4. \#12, 241 (1984)

2. R.D. MacElroy and J. Bredt. Current concepts and future directions of CELSS. Adv. Space Res. 4. \#12. 221 (1984)

3. M.M. Averner. An approach to the mathematical modelling of a controlled ecological life support system. NASA CR-166331 (1981).

4. P.S. Babcock. D.M. Auslander. and R.C. Spear. Dynamic considerations for control of closed life support systems. Adv. Space Res. 4. \#12. 263 (1984)

5. J.D. Stahr, D.M. Auslander, R.C. Spear, and G.E. Young. An Approach to the preliminary evaluation of Closed Ecological Life Support System (CELSS) scenarios and control strategies. NASA CR-166368 (1982)

6. E.P. Odum. Fundamentals of Ecology (Third Edition). W.B. Saunders, Philadelphia, 1971.

7. S.P. Columbano, private communication (1985)

8. T. Volk and J.D. Rummel, this issue.

9. H.T. Odum. Systems Ecology: An Introduction, John Wiley. New York. 1983.

10. F.B. Salisbury and B.G. Bugbee, private communication (1986)

11. Anonymous. Trade-off study and conceptual designs of regenerative advanced integrated life support systems (AILSS). NASA CR-1458 (1970)

12. Anonymous. Space station reference configuration description. NASA JSC-19989 (1984)

13. C.W. Miller, D.B. Heppner. Space station environmental control/life support system engineering. SAE Paper No. 851375. San Francisco (1985)

14. S.H. Timberlake. G.T. Hong. M. Simson. and M. Modell, Supercritical water oxidation for wastewater treatment: preliminary study of urea destruction. SAE Paper 820872 (1982)

15. M. Oleson. T. Slavin, and F. Liening. Controlled Ecological Life Support System (CELSS) physicochemical waste management systems evaluation. NASA CR-177422 (1986) 